\title{
A Self-Dual Yang-Mills Hierarchy and its Reductions to Integrable Systems in $1+1$ and $2+1$ Dimensions
}

\author{
Mark J. Ablowitz ${ }^{1}$, Sarbarish Chakravarty ${ }^{1}$, and Leon A. Takhtajan ${ }^{2}$ \\ ${ }^{1}$ Program in Applied Mathematics, University of Colorado, Boulder, CO 80309-0526, USA \\ 2 Department of Mathematics, State University of New York at Stony Brook, Stony Brook, N.Y. \\ 11794-3651, USA
}

Received September 1, 1992

\begin{abstract}
The self-dual Yang-Mills equations play a central role in the study of integrable systems. In this paper we develop a formalism for deriving a four dimensional integrable hierarchy of commuting nonlinear flows containing the self-dual Yang-Mills flow as the first member. We show that upon appropriate reduction and suitable choice of gauge group it produces virtually all well known hierarchies of soliton equations in $1+1$ and $2+1$ dimensions and can be considered as a "universal" integrable hierarchy. Prototypical examples of reductions to classical soliton equations are presented and related issues such as recursion operators, symmetries, and conservation laws are discussed.
\end{abstract}

\section{Introduction}

In recent years there has been considerable interest in the self-dual Yang-Mills (SDYM) equations. Originated from the non-perturbative approach to the quantum theory of gauge fields [1], SDYM system became useful also in general relativity [2]; in mathematics it was a major tool for achieving dramatic progress in the theory of four-manifolds [3]. Moreover, SDYM equations turn out to be an integrable system in the sense that associated with them there exists a compatible pair of linear PDE's from which significant information about the SDYM equations can be deduced, e.g. solutions via the Riemann-Hilbert factorization problem, Bäcklund transformations, conservation laws, hierarchies, etc. [4, a, b, c].

More recently, many well known soliton equations solvable by the inverse scattering transform (IST) have been found as reductions of the SDYM system which has led to the conjecture that perhaps it contains "all" integrable soliton systems [5]. Indeed by extending the notion of SDYM system to encompass the higher flows we give more credence to this possibility. In particular, we show that the SDYM equations can be embedded naturally in a hierarchy of integrable equations of which they can be considered as the principal starting member. Significantly, the classical soliton hierarchies are constructed in a similar way and to stress this point further we show how the resulting four dimensional "universal" hierarchy reduces to the standard $1+1$ dimensional soliton hierarchies. In order 
to incorporate the $2+1$ hierarchies into the basic formalism as well we introduce a SDYM system with a suitable choice of infinite dimensional gauge group. Examples discussed include the $N$-wave equations in $1+1$ and $2+1$ dimensions, the Korteweg-de Vries (KdV) and Kadomtsev-Petviashvili (KP) equations, and the nonlinear Schrödinger (NLS) and Davey-Stewartson (D-S) equations and their associated hierarchies.

The paper is organized as follows. In Sect. 2 we briefly review the basic background information for the SDYM equations on $\mathbb{R}^{4}$. In Sect. 3 we derive a four dimensional hierarchy associated with the SDYM equations. Our approach is based upon the concept of intertwining operators and uses algebraic analysis; we treat these operators as formal series in the "spectral" parameter appearing in the linear system of the SDYM equations. We also discuss the recursion operators, symmetries and conservation laws associated with this hierarchy. In Sect. 4 we illustrate how to obtain the hierarchies and local conservation laws for classical $1+1$ soliton equations as reductions of the SDYM hierarchy. In Sect. 5 we show that by considering the SDYM system with an infinite dimensional gauge algebra of formal pseudodifferential operators it is possible to treat $2+1$ dimensional integrable equations and their hierarchies as a reductions of our formalism.

While we have taken a rather formal point of view in the text of this paper we have added numerous explanatory remarks which are also intended to describe different aspects of the "universality" of the SDYM system. We have also indicated different methods to prove a result whenever we felt that it would be beneficial for the reader.

\section{SDYM as an Integrable System}

In this section we briefly recall the basic definitions and properties of the SDYM equations (see [6] and references therein). Formally speaking these are equations for the self-dual unitary connection in a Hermitian vector bundle $E$ associated with the principal $G$-bundle over a Riemannian four-manifold $M$. The corresponding Lie group $G$ is called the gauge group and its Lie algebra $g$ - the gauge algebra. Introducing the curvature form $F=d A+A \wedge A$ of the connection $A$ and the Hodge star-operator $*$, we can write SDYM equations as

$$
F=* F \text {. }
$$

The SDYM equations are gauge invariant, i.e. the self-duality property (1) is preserved under the gauge transformations $A \mapsto A^{f}=f A f^{-1}+d f f^{-1}$, where $f \in \operatorname{Map}(M, G)$.

Leaving aside the global considerations, we will assume here that $E$ is a trivial vector bundle over the Euclidean space $\mathbb{R}^{4}$ with fibers isomorphic as linear spaces to the Lie algebra $g$. In standard coordinates $x=\left(x^{\mu}\right), \mu=0,1,2,3$, on $M=\mathbb{R}^{4}$ the connection $A$ and curvature $F$ are realized by the following $g$-valued differential forms: $A=\sum_{\mu=0}^{3} A_{\mu}(\mathbf{x}) d x^{\mu}, F=\sum_{\mu, v=0}^{3} F_{\mu \nu}(\mathbf{x}) d x^{\mu} \wedge d x^{\nu}$, where

$$
F_{\mu \nu}=\partial_{\mu} A_{v}-\partial_{v} A_{\mu}-\left[A_{\mu}, A_{v}\right] \text {. }
$$

The $g$-valued coefficients $A_{\mu}(\mathbf{x})$ and $F_{\mu \nu}(\mathbf{x})$ are called Yang-Mills potentials and Yang-Mills fields respectively. Equation (1) can be written down explicitly as the 
following nonlinear system of first order PDE's:

$$
F_{01}=F_{23}, \quad F_{02}=F_{31}, \quad F_{03}=F_{12}
$$

for the Yang-Mills potentials $A_{\mu}$ (defined up to a gauge transformations $A_{\mu} \mapsto A_{\mu}^{f}=f A_{\mu} f^{-1}+\partial_{\mu} f f^{-1}$ ).

The SDYM equations have the important property that they result from the compatibility condition of a certain auxiliary linear problem $[7,8]$. Namely, introducing on $\mathbb{R}^{4}$ the complex coordinates $y=x^{1}+i x^{2}, \quad \bar{y}=x^{1}-i x^{2}$, $z=x^{0}+i x^{3}, \bar{z}=x^{0}-i x^{3}$, the SDYM equations can be rewritten as

$$
F_{y z}=F_{\bar{y} \bar{z}}=0, \quad F_{y \bar{y}}+F_{z \bar{z}}=0
$$

and are the compatibility condition for the following linear system:

$$
\begin{aligned}
& D_{1} \Psi=A_{1} \Psi, \\
& D_{2} \Psi=A_{2} \Psi,
\end{aligned}
$$

where

$$
\begin{array}{ll}
D_{1}=\partial_{y}+\lambda \partial_{\bar{z}}, & A_{1}=A_{y}+\lambda A_{\bar{z}}, \\
D_{2}=\partial_{z}-\lambda \partial_{\bar{y}}, & A_{2}=A_{z}-\lambda A_{\bar{y}},
\end{array}
$$

and the variable $\lambda \in \mathbf{P}^{1}=\mathbb{C} \cup\{\infty\}$ plays the role of the spectral parameter. Indeed, the compatibility for this system (for all values of $\lambda$ ) has the form

$$
D_{2} A_{1}-D_{1} A_{2}+\left[A_{1}, A_{2}\right]=0,
$$

and yields Eqs. (3) as vanishing coefficients of the quadratic polynomial in $\lambda$ in (6).

The representation (6) indicates that SDYM equations are "exactly solvable." More precisely, using the auxiliary system (4)-(5) it is possible to construct SDYM solutions with the help of a Riemann-Hilbert factorization problem [8]. Namely, let $A_{\mu}(\mathbf{x})$ be a solution of (3) and $\Psi_{0}(\mathbf{x}, \lambda)$ and $\Psi_{\infty}(\mathbf{x}, \lambda)$ be two $G$-valued solutions of (4)-(5), holomorphic in the neighborhoods $U_{0}$ of 0 and $U_{\infty}$ of $\infty$ in $\mathbf{P}^{1}$ respectively. Then the "ratio"

$$
\Psi=\Psi_{\infty}^{-1} \Psi_{0}
$$

satisfies, in a common domain of analyticity $U_{0} \cap U_{\infty}$, the equations

$$
D_{1} \Psi=D_{2} \Psi=0
$$

so that

$$
\Psi(\mathbf{x}, \lambda)=S(\lambda y-\bar{z}, \lambda z+\bar{y}, \lambda)
$$

is a $G$-valued holomorphic function. Conversely, let $U_{0}$ and $U_{\infty}$ be the covering of $\mathbf{P}^{1}$ and $S$ be the function above. Define $\Psi$ by (8) and consider (7) as a family of Riemann-Hilbert factorization problems, parametrized by $y, \bar{y}, z, \bar{z}$, for determining $G$-valued functions $\Psi_{0}, \Psi_{\infty}$ from a given $\Psi$ defined in the intersection $U_{0} \cap U_{\infty}$. Then functions $\Psi_{0}, \Psi_{\infty}$ satisfy, with proper $\lambda$-dependence, the linear system (4)-(5) and the corresponding Yang-Mills potentials $A_{\mu}(\mathbf{x})$ obtained from $D_{1,2} \Psi_{0} \Psi_{0}^{-1}=D_{1,2} \Psi_{\infty} \Psi_{\infty}^{-1}$ satisfy the SDYM equations.

With suitable conditions [8] a solution of the Riemann-Hilbert problem (7) exists; subject to a proper normalization it has a unique solution. Different normalizations lead to the left action of $\operatorname{Map}\left(\mathbb{R}^{4}, G\right)$ on $\Psi_{0}, \Psi_{\infty}$ and give rise to gauge equivalent Yang-Mills potentials $A_{\mu}$. 


\section{SDYM Hierarchy}

It is significant that the SDYM equations share many common properties with $1+1$ dimensional integrable solitonic equations. In this section we will show that there exists a hierarchy of "commuting higher SDYM flows" (cf. the well known $\mathrm{KdV}$ hierarchy) with related symmetries and recursion operator.

We start our construction by choosing a convenient gauge.

Lemma 1. By a suitable gauge transformation any SDYM connection admits the form where the components $A_{\bar{y}}$ and $A_{\bar{z}}$ are represented by two arbitrary $\mathbf{x}$-independent commuting elements in $g$ so that in this gauge the SDYM system reduces to

$$
F_{y z}=0, \quad F_{y \bar{y}}+F_{z \bar{z}}=0 .
$$

Proof. Equation $F_{\bar{y} \bar{z}}=0$ implies that connection $A$ has a zero curvature in the $\bar{y} \bar{z}$-plane, so that the Yang-Mills potentials $A_{\bar{y}}$ and $A_{\bar{z}}$ are (locally) "pure gauge": there exists $f_{0} \in \operatorname{Map}\left(\mathbb{R}^{4}, G\right)$ such that $A_{\bar{y}}=\partial_{\bar{y}} f_{0} f_{0}^{-1}, A_{\bar{z}}=\partial_{\bar{z}} f_{0} f_{0}^{-1}$. Therefore under the gauge transformation $f=\exp \left(\bar{y} a_{1}+\bar{z} a_{2}\right) f_{0}^{-1}$, where $a_{1}, a_{2} \in g$, $\left[a_{1}, a_{2}\right]=0, A \frac{f}{y}=a_{1}, A \frac{f}{2}=a_{2}$.

We will use this particular gauge choice throughout the paper unless otherwise stated explicitly. This choice makes it possible to treat the coordinates $y, \bar{y}, \bar{z}$, as the "spatial" variables and $z$ - as the "time" variable.

Our approach to the SDYM hierarchy is based on the general concept of intertwining operators which goes back to Schur [9] (see discussion in [10]) and was introduced into the theory of integrable systems by Sato [11] and extensively used thereafter (see, e.g., [12] and references therein). The intertwiners transform "bare unperturbed" auxiliary linear systems into "perturbed" ones and according to the rules of algebraic analysis we will treat them as formal power series in $\lambda^{-1}$. Specifically for our problem these intertwiners will be nonlocal functionals of the Yang-Mills potentials. Therefore we introduce the following notation.

Denote by $\hat{g}$ the Lie algebra of $g$-valued functions $\mathbb{R}^{4}$ and their "primitives" with respect to the following first order differential operator with constant coefficients: $\partial_{\bar{z}}-\operatorname{ad} A_{\bar{z}}$, where ad is adjoint $g$-action: $\operatorname{ad} A_{\bar{z}}(B)=\left[A_{\bar{z}}, B\right]$. In other words, elements in $\hat{g}$ consist of polynomials of the Yang-Mills potentials and their partial derivatives together with nonlocal expressions obtained by successive applications of the inverse of $\partial_{\bar{z}}-\operatorname{ad} A_{\bar{z}}$ to such functions. One can pass from formal to rigorous theory provided the action of $\left(\partial_{\bar{z}}-\operatorname{ad} A_{\bar{z}}\right)^{-1}$ is well defined in certain functional classes (say periodic or rapidly decreasing). Furthermore, let

$$
\hat{g}\left(\left(\lambda^{-1}\right)\right)=\sum_{k=-\infty}^{k \ll \infty} \oplus \hat{g} \lambda^{k}
$$

be the Lie algebra of formal semi-infinite (finite at $+\infty$ ) Laurent series in $\lambda$ with $\hat{g}$-valued coefficients and " + " and " - " be the complementary projectors on the polynomial part: $\left.\hat{g}\right|_{+}=\sum_{0 \leqq k \leqslant \infty} \oplus \hat{g} \lambda^{k}$ and the "pure Laurent" part: $\left.\hat{g}\right|_{-}$ $=\sum_{k \leqq-1} \oplus \hat{g} \lambda^{k}$ in $\hat{g}\left(\left(\lambda^{-1}\right)\right)$ respectively. Finally, denote by $\hat{G} \mid$ - the Lie group corresponding to the Lie algebra $\left.\hat{g}\right|_{-}$.

Lemma 2. There exists $W(\mathbf{x}, \lambda) \in \hat{G} \mid$ _ such that the corresponding multiplication operator $\hat{W}$ is the intertwiner for the SDYM linear problem, i.e.

$$
D_{1}-A_{1}=\hat{W}\left(D_{1}-\lambda A_{\bar{z}}\right) \hat{W}^{-1} \text {. }
$$


Proof. Rewrite (10) in the form

$$
D_{1} W W^{-1}=A_{1}-\lambda W A_{\bar{z}} W^{-1}
$$

and look for the expansions

$$
D_{1} W W^{-1}=\sum_{n=0}^{\infty} \frac{V_{n}(\mathbf{x})}{\lambda^{n}}, \quad W A_{\bar{z}} W^{-1}=A_{\bar{z}}+\sum_{n=1}^{\infty} \frac{W_{n}(\mathbf{x})}{\lambda^{n}},
$$

where $V_{n}, W_{n} \in \hat{g}$. Substituting them into Eq. (11) we obtain $W_{1}=A_{y}-V_{0}$, $W_{n}=-V_{n-1}, n>1$. Now consider the differential equation

$$
D_{1}\left(W A_{\bar{z}} W^{-1}\right)=\left[D_{1} W W^{-1}, W A_{\bar{z}} W^{-1}\right]=\left[A_{1}, W A_{\bar{z}} W^{-1}\right]
$$

which produces the recurrence relation for $W_{n}$

$$
\left(\partial_{\bar{z}}-\operatorname{ad} A_{\bar{z}}\right) W_{n+1}=-\left(\partial_{y}-\operatorname{ad} A_{y}\right) W_{n}, \quad n \geqq 1,
$$

with the initial condition

$$
\left(\partial_{\bar{z}}-\operatorname{ad} A_{\bar{z}}\right) W_{1}=\left[A_{y}, A_{\bar{z}}\right] .
$$

Hence coefficients $W_{n}$ and therefore, $V_{n}$, can be determined recursively and the existence of $W(\mathbf{x}, \lambda) \in \hat{G} \mid$ - follows from the differential equation (11) via the "exponential map" since the right-hand side in (11) belongs to $\left.\hat{g}\right|_{-}$.

Remark 1. Equation (11) reads

$$
A_{1}=D_{1} W W^{-1}+\lambda W A_{\bar{z}} W^{-1},
$$

so that $W(\mathbf{x}, \lambda)$ has a meaning of a formal ( $\lambda$-dependent!) gauge transformation relating "unperturbed" and "perturbed" Yang-Mills potentials $\lambda A_{\bar{z}}$ and $A_{1}$.

Remark 2. There exists another proof of Lemma 2 for the case when the gauge group $G=G L(n)$. Namely, let $g=n_{+} \oplus h \oplus n_{-}$be the Cartan decomposition of the Lie algebra $g=g l(n)$. Assuming that $A_{\bar{y}}, A_{\bar{z}} \in h$ (i.e. are diagonal matrices) we have the following representation for the intertwiner $W(\mathbf{x}, \lambda)$ :

$$
W=(\mathbf{1}+M) \exp Z,
$$

where 1 stands for the unit matrix, $M(\mathbf{x}, \lambda) \in \hat{n})_{-}\left(n=n_{+} \oplus n_{-}\right.$is the nilpotent subspace in $g$ ) and $\left.Z(\mathbf{x}, \lambda) \in \hat{h}\right|_{-}$(in other words, $M$ and $Z$ are off diagonal and diagonal matrices respectively). Indeed, substituting representation (14) into Eq. (11) and "separating diagonal and off diagonal parts" we obtain the following system

$$
\begin{aligned}
D_{1} M & =\left(A_{y}+A_{y} M\right)_{n}-M\left(A_{y}+A_{y} M\right)_{c}+\lambda\left[A_{\bar{z}}, M\right], \\
D_{1} Z & =\left(A_{y}+A_{y} M\right)_{c},
\end{aligned}
$$

where the subscripts " $n$ " and " $c$ " denote the projectors onto nilpotent and Cartan subspaces respectively. Now consider the expansions

$$
M=\sum_{k=1}^{\infty} \frac{M_{k}(\mathbf{x})}{\lambda^{k}}, \quad Z=\sum_{k=1}^{\infty} \frac{Z_{k}(\mathbf{x})}{\lambda^{k}}
$$


and substitute them into the system (15)-(16). We obtain the following recurrence relations

$$
\begin{aligned}
\left(\partial_{\bar{z}}-\operatorname{ad} A_{\bar{z}}\right) M_{k+1} & =-\partial_{y} M_{k}+\left(A_{y} M_{k}\right)_{n}-M_{k}\left(A_{y}\right)_{c}-\sum_{l=1}^{k-1} M_{k}\left(A_{y} M_{k-l}\right)_{c}, \\
\partial_{\bar{z}} Z_{k+1} & =-\partial_{y} Z_{k}+\left(A_{y} M_{k}\right)_{c}, \quad k>1,
\end{aligned}
$$

with initial conditions

$$
\left(\partial_{\bar{z}}-\operatorname{ad} A_{\bar{z}}\right) M_{1}=\left(A_{y}\right)_{n}, \quad \partial_{\bar{z}} Z_{1}=\left(A_{y}\right)_{c}
$$

for determining the coefficients $M_{k}, Z_{k}$ (cf. [12]).

Remark 3. Define $\Psi=W \Psi_{0}$, where $\Psi_{0}$ satisfies the "unperturbed" part of Eq. (4) with $A_{y}=0$, i.e. $D_{1} \Psi_{0}=\lambda A_{\bar{z}} \Psi_{0}$, and $W$ is given by Lemma 2 . Then $\Psi$ formally satisfies (4) with $A_{1}=A_{y}+\lambda A_{\bar{z}}$ and can be considered as a "WKB solution" of (4) as $\lambda \rightarrow \infty$. For a certain class of boundary conditions (e.g. rapidly decreasing or periodic cases) $\Psi(\mathbf{x}, \lambda)$ is indeed an asymptotic solution of (4).

Remark 4. If, in addition, $\Psi_{0}$ satisfies (5) with $A_{z}=0$, i.e. $D_{2} \Psi_{0}=-\lambda A_{\bar{y}} \Psi_{0}$, and $\hat{W}$ also intertwines $D_{2}-A_{2}$ and $D_{2}+\lambda A_{\bar{y}}$, i.e. $D_{2}-A_{2}=\hat{W}\left(D_{2}+\lambda A_{\bar{y}}\right) \hat{W}^{-1}$, then $\Psi$ also satisfies Eq. (5) with $A_{2}=A_{z}-\lambda A_{\bar{y}}$. This is the essence of the "dressing procedure" which starts from the "bare" SDYM solutions: $A_{y}=A_{z}=0, A_{\bar{y}}$ and $A_{\bar{z}}$ are commuting constants, and yields a new SDYM solution $A_{y}, A_{z}$ (with the same gauge choice for $A_{\bar{y}}$ and $\left.A_{\bar{z}}\right)$. $\left[D_{1}-\lambda A_{\bar{z}}, D_{2}+\lambda A_{\bar{y}}\right]=0$ is valid by construction (commuting $A_{\bar{y}}$ and $A_{\bar{z}}$ ) whereas $\left[D_{1}-A_{1}, D_{2}-A_{2}\right]=0$ is due to Lemma 2.

Next set

$$
\partial_{\bar{y}}+\hat{\Phi}=\hat{W}\left(\partial_{\bar{y}}-A_{\bar{y}}\right) \hat{W}^{-1},
$$

or

$$
\Phi=W\left(\partial_{\bar{y}}-A_{\bar{y}}\right) W^{-1}=-\partial_{\bar{y}} W W^{-1}-W A_{\bar{y}} W^{-1}=\sum_{n=0}^{\infty} \frac{\Phi_{n}}{\lambda^{n}}, \quad \Phi_{n} \in \hat{g},
$$

where $\Phi_{0}=-A_{\bar{y}}$ and $W$ is given by Lemma 2 .

Lemma 3. Coefficients $\Phi_{n} \in \hat{g}, n \geqq 1$, are uniquely determined by the initial condition $\Phi_{0}=-A_{\bar{y}}$.

Proof. We have $\partial_{\bar{y}}+\hat{\Phi}=\hat{W}\left(\partial_{\bar{y}}-A_{\bar{y}}\right) \hat{W}^{-1}$, so that by the intertwining property

$$
\left[D_{1}-A_{1}, \partial_{\bar{y}}+\hat{\Phi}\right]=\hat{W}\left[D_{1}-\lambda A_{\bar{z}}, \partial_{\bar{y}}-A_{\bar{y}}\right] \hat{W}^{-1}=0,
$$

which yields the differential equation for $\Phi$ :

$$
D_{1} \Phi=\left[A_{1}, \Phi\right]-\partial_{\bar{y}} A_{1} .
$$

Substituting into it the expansion for $\Phi$, we get that the linear term in $\lambda$ vanishes identically because of $\Phi_{0}=-A_{\bar{y}}$ and commutativity of $A_{\bar{y}}$ and $A_{\bar{z}}$, whereas vanishing of the non-positive powers of $\lambda$ lead to a recurrence relation

$$
\left(\partial_{\bar{z}}-\operatorname{ad} A_{\bar{z}}\right) \Phi_{n+1}=-\left(\partial_{y}-\operatorname{ad} A_{y}\right) \Phi_{n}-\partial_{\bar{y}} A_{y} \delta_{n 0}, \quad n \geqq 0 .
$$


Notice that all constructions in Lemmas 2-3 involve only the values of the Yang-Mills potentials at a "time slice" $z=$ constant, i.e. for fixed $z$. This important property allows us to define naturally a SDYM hierarchy by introducing infinitely many "times" $z_{k}, k=2, \ldots$, where $z_{2}=z$ is the usual SDYM "time." Namely, set

$$
D_{k}=\partial_{z_{k}}-\lambda^{k-1} \partial_{\bar{y}}, \quad A_{k}=\left(\lambda^{k-1} \Phi\right)_{+}, \quad k \geqq 2 .
$$

Definition 1. A SDYM hierarchy is defined by the infinite sequence of flows with respect to the "times" $z_{k}$, with the $k^{\text {th }}$ member given by the equation

$$
\left[D_{1}-A_{1}, D_{k}-A_{k}\right]=0, \quad k \geqq 2,
$$

i.e.

$$
D_{k} A_{1}-D_{1} A_{k}+\left[A_{1}, A_{k}\right]=0,
$$

which arises as a compatibility condition for the linear system

$$
\begin{aligned}
& D_{1} \Psi=A_{1} \Psi, \\
& D_{k} \Psi=A_{k} \Psi .
\end{aligned}
$$

Remark 5. For $k=2$, we have $A_{2}=(\lambda \Phi)_{+}=\Phi_{1}+\lambda \Phi_{0}=A_{z}-\lambda A_{\bar{y}}$ since Eq. (19) for $n=1$, which determines $\Phi_{1}$, coincides with the second SDYM equation in (9) if one identifies $\Phi_{1}$ and $A_{z}$. The compatibility condition yields $F_{y z}=0$ - the remaining equation in the SDYM system (9) for the "time" variable $z_{2}=z$. The higher SDYM flows are given explicitly by the equations of motion (the $k^{\text {th }}$ flow)

$$
\partial_{z_{k}} A_{y}-\partial_{y} \Phi_{k-1}+\left[A_{y}, \Phi_{k-1}\right]=0,
$$

where $\Phi_{k-1}$ are defined recursively by (19) and contain (for $k>2$ ) the inverse powers of $\partial_{\bar{z}}-\operatorname{ad} A_{\bar{z}}$; they are, therefore, nonlocal.

Remark 6. Using recurrence relation (19), we have for $k \geqq 1$

$$
\Phi_{k+1}=R \Phi_{k},
$$

where $R=-\left(\partial_{\bar{z}}-\operatorname{ad} A_{\bar{z}}\right)^{-1}\left(\partial_{y}-\operatorname{ad} A_{y}\right)$ and may be considered as a recursion operator for the SDYM hierarchy. Although this definition is formal (the inverse should be properly defined), under SDYM reductions $R$ yields well known (and well defined) recursion operators for integrable systems.

Remark 7. The $k^{\text {th }}$ flow in the SDYM hierarchy can be considered as a "dressing" of a "bare" solution by the intertwiner $W$, i.e.

$$
\begin{aligned}
D_{1}-A_{1} & =\hat{W}\left(D_{1}-\lambda A_{\bar{z}}\right) \hat{W}^{-1}, \\
D_{k}-A_{k} & =\hat{W}\left(D_{k}+\lambda^{k-1} A_{\bar{y}}\right) \hat{W}^{-1} .
\end{aligned}
$$

The latter equation implies

$$
A_{k}=\partial_{z_{k}} W W^{-1}-\lambda^{k-1}\left(\partial_{\bar{y}} W W^{-1}+W A_{\bar{y}} W^{-1}\right)=\partial_{z_{k}} W W^{-1}+\lambda^{k-1} \Phi .
$$

Since $A_{k}=\left(\lambda^{k-1} \Phi\right)_{+}$and $\left.\partial_{z_{k}} W W^{-1} \in \hat{g}\right|_{-}$, it yields the $z_{k}$-evolution of the intertwiner $W$ :

$$
\partial_{z_{k}} W W^{-1}=-\left(\lambda^{k-1} \Phi\right)_{-} .
$$

The corresponding evolution equation for $\Phi$ is given by

$$
D_{k} \Phi=\left[A_{k}, \Phi\right]-\partial_{\bar{y}} A_{k},
$$


since, by the definition of $A_{k}, \Phi$ and the intertwining property,

$$
\left[D_{k}-A_{k}, \partial_{\bar{y}}+\hat{\Phi}\right]=\hat{W}\left[D_{k}+\lambda^{k-1} A_{\bar{y}}, \partial_{\bar{y}}-A_{\bar{y}}\right] \hat{W}^{-1}=0 .
$$

Theorem 1. The SDYM hierarchy consists of commuting flows, i.e. there exists a common intertwiner $\hat{W}$ for all higher SDYM equations.

Proof. It is sufficient to show that there exists a common solution to the following infinite system of equations:

$$
\partial_{z_{k}} W=U_{-}^{(k)} W, \quad k \geqq 2,
$$

where $U^{(k)}=-\lambda^{k-1} \Phi$ (see Remark 7). Indeed, the compatibility condition for (23) reads

$$
\partial_{z_{l}} U^{(k)}-\partial_{z_{k}} U^{(l)}+\left[U^{(k)}, U^{(l)}\right]=0
$$

and should be valid for all $k, l \geqq 2$. Multiplying Eqs. (22) for the "times" $z_{k}$ and $z_{l}$ by $\lambda^{k-1}$ and $\lambda^{l-1}$ respectively, subtracting and finally projecting the difference onto $\hat{g} \mid$ - we get that

$$
\partial_{z_{l}} U_{-}^{(k)}-\partial_{z_{k}} U^{(l)}=\left[A_{l}, U^{(k)}\right]_{-}-\left[A_{k}, U^{(l)}\right]_{-} .
$$

Thus we can rewrite the left-hand side of (24) by using this last equation, the formulas $A_{k}=-U_{+}^{(k)}, A_{l}=-U_{+}^{(l)}$ and the fact that $U^{(k)}$ and $U^{(l)}$ commute:

$$
-\left[U_{+}^{(l)}, U^{(k)}\right]_{-}+\left[U_{+}^{(k)}, U^{(l)}\right]_{-}+\left[U_{-}^{(k)}, U_{-}^{(l)}\right]=\left[U^{(k)}, U^{(l)}\right]_{-}=0,
$$

which completes the proof.

Remark 8. This proof can be considered as a "universal proof" of commutativity for all hierarchies of integrable equations, i.e. it covers all possible SDYM reductions including the well-known cases of $N$-wave, NLS, KdV and KP hierarchies.

Remark 9. Let $\Psi_{0}$ be the common solution of the following (obviously compatible) "bare" system of equations:

$$
\begin{aligned}
& D_{1} \Psi_{0}=\lambda A_{\bar{z}} \Psi_{0}, \\
& D_{k} \Psi_{0}=-\lambda^{k-1} A_{\bar{y}} \Psi_{0}, \quad k \geqq 2 .
\end{aligned}
$$

Then $\Psi=W \Psi_{0}$ is the common solution of the following "dressed" system

$$
D_{k} \Psi=A_{k} \Psi, \quad k \geqq 1 .
$$

In other words, $\Psi\left(y, \bar{y}, \bar{z}, z_{2}, \ldots\right)$ is a common (formal) "wave-function" for the entire SDYM hierarchy.

Remark 10. It follows from the definition of the SDYM hierarchy that its two successive flows are related by

$$
\left(D_{k+1}-A_{k+1}\right)-\lambda\left(D_{k}-A_{k}\right)=\partial_{z_{k+1}}-\lambda \partial_{z_{k}}-\Phi_{k},
$$

where the right-hand side is linear in $\lambda$. Due to the Theorem $1\left[D_{1}-A_{1}\right.$, $\left.\partial_{z_{k+1}}-\lambda \partial_{z_{k}}-\Phi_{k}\right]=0$, so that we have a compatible linear system

$$
\begin{aligned}
D_{1} \Psi & =A_{1} \Psi, \\
\left(\partial_{z_{k+1}}-\lambda \partial_{z_{k}}\right) \Psi & =\Phi_{k} \Psi,
\end{aligned}
$$


which is similar to the SDYM original system (4)-(5). In fact, if we introduce the auxiliary space-time $\mathbb{C}^{4}$ with coordinates $y, \bar{z}, z_{k}, z_{k+1}$, then $A_{y}, A_{\bar{z}}, 0, \Phi_{k}$ play the role of Yang-Mills potentials and satisfy the complexified SDYM equations in $\mathbb{C}^{4}$. Thus there exists an infinite set of complexified SDYM connections embedded into the SDYM hierarchy. Conversely, it is possible to reconstruct the SDYM hierarchy by considering appropriate linear combinations of the infinite set of SDYM connections (whose "space-time manifolds" have common two-dimensional part). A similar approach was used in [13] to obtain the KdV and NLS hierarchies from the Bogomolny equations.

Remark 11. The gauge transformation $A \mapsto A^{f}, f \in \operatorname{Map}\left(\mathbb{R}^{4}, G\right)$ (see Sect. 2) results in the left action on the intertwiner $W \mapsto W^{f}=f W$ and (since $f$ is $\lambda$ independent) in the gauge action on $\Phi: \Phi \mapsto \Phi^{f}=-\partial_{\bar{y}} f f^{-1}+f \Phi f^{-1}$. This remark will be useful in the next section, while discussing the KdV equation, where we use a gauge choice different from that in Lemma 1.

Now let us consider symmetries and conservation laws for the SDYM hierarchy.

Definition 2. A symmetry $\left\{\delta A_{k}\right\}_{k=1}^{\infty}$ associated with the solution $\left\{A_{k}\right\}_{k=1}^{\infty}$ of the $S D Y M$ hierarchy is a solution of the linearized equations of motion, i.e.

$$
D_{k} \delta A_{l}-D_{l} \delta A_{k}+\left[\delta A_{l}, A_{k}\right]+\left[A_{l}, \delta A_{k}\right]=0, \quad k, l \geqq 1 .
$$

In other words, symmetries are tangent vectors to the (infinite dimensional) solution manifold A of the SDYM hierarchy, i.e. they are vector fields on $\mathbf{A}$. (We note that the "variations" $\delta A_{k}$ are polynomials in $\lambda$ of one order less than that of $A_{k}$ since $\delta A_{\bar{y}}=\delta A_{\bar{z}}=0$.)

Let $\left\{A_{k}\right\}_{k=1}^{\infty}$ be the solution of the SDYM hierarchy and $\left\{\delta A_{k}\right\}_{k=1}^{\infty}$ be the symmetry with given $\delta A_{1}=\delta A_{y}$. Denote by $\delta W$ the linearized ("infinitesimal") intertwiner $W$ and set $J=\delta W W^{-1}$.

Theorem 2. i) The element $\left.J \in \hat{g}\right|_{-}$,

$$
J=\left.\sum_{n=1}^{\infty} \frac{J_{n}}{\lambda^{n}} \in \hat{g}\right|_{-},
$$

is uniquely determined by a given $\delta A_{y}$ :

$$
J_{n}=R^{n-1} J_{1}, \quad n \geqq 1, \quad\left(\partial_{\bar{z}}-\operatorname{ad} A_{\bar{z}}\right) J_{1}=\delta A_{y} .
$$

ii) The "time"-dependence of $J$ is given by the equations

$$
\partial_{z_{k}} J=\left(\lambda^{k-1} \partial_{\bar{y}} J+\left[A_{k}, J\right]\right)_{-} .
$$

iii) The symmetries $\delta A_{k}$ admit the representation

$$
\delta A_{k}=D_{k} J-\left[A_{k}, J\right], \quad k \geqq 1 .
$$

Proof. Linearization of the "dressing" formulas (see Remark 7) immediately proves iii); the "- "-component of iii) yields the evolution equations in ii). In order to prove i), consider the equation in iii) for $k=1$, i.e. $\delta A_{y}=D_{1} J-\left[A_{1}, J\right]$, and expand it in negative powers of $\lambda$.

Remark 12. Due to Theorem 2 any choice for $J$ produces symmetries. A particular family of symmetries can be obtained by a special choice for $J$ given by

$$
J_{1}^{(k)}=\Phi_{k}, \quad k \geqq 1 .
$$


Comparing Lemma 3 and Theorem 2 we see that in this case it is possible to solve recurrence relations for $J_{n}^{(k)}$ explicitly:

$$
J_{n}^{(k)}=\Phi_{n+k-1}
$$

so that for the generating function $J^{(k)}=\sum_{n=1}^{\infty} J_{n}^{(k)} \lambda^{-n}$ of this family we get $J^{(k)}=\left(\lambda^{k-1} \Phi\right)_{-}=-U_{-}^{(k)}$. These symmetries for the SDYM hierarchy are the extension of those introduced in [14] for the SDYM system $(k=2)$.

Now let $K$ be a common solution of the equations $D_{k} K=0, k \geqq 1$, then $I=W K W^{-1}$ satisfies

$$
D_{k} I=\left[A_{k}, I\right] .
$$

Assume, in addition, that on the Lie algebra $\hat{g}\left(\left(\lambda^{-1}\right)\right)$ there exists an invariant bilinear form $($,$) and define I_{k}=\left(I, \delta A_{k}\right)$.

Theorem 3. The family $\left\{I_{k}\right\}_{k=1}^{\infty}$ for an arbitrary "starting" symmetry $\delta A_{y}$ gives a set of conservation laws for the SDYM hierarchy, i.e.

$$
D_{k} I_{l}=D_{l} I_{k}, \quad k, l \geqq 1 .
$$

Proof. We have

$$
D_{k} I_{l}=D_{k}\left(I, \delta A_{l}\right)=\left(\left[A_{k}, I\right], \delta A_{l}\right)+\left(I, D_{k} \delta A_{l}\right),
$$

or, using the definition of symmetries and the invariance of $($,$) ,$

$$
\begin{aligned}
D_{k} I_{l} & =\left(\left[A_{k}, I\right], \delta A_{l}\right)+\left(I, D_{l} \delta A_{k}+\left[\delta A_{k}, A_{l}\right]+\left[A_{k}, \delta A_{l}\right]\right) \\
& =\left(I, D_{l} \delta A_{k}\right)+\left(\left[A_{l}, I\right], \delta A_{k}\right)=D_{l}\left(I, \delta A_{k}\right)=D_{l} I_{k} .
\end{aligned}
$$

The following two remarks will be useful in deriving the standard conservation laws for $1+1$ integrable systems.

Remark 13. Another construction of conservation laws is based on the operator $\partial_{\lambda}$. Namely, consider the dressing equations from Remark 7 and differentiate them with respect to $\lambda$. Introducing $J=\partial_{\lambda} W W^{-1}$ we get that

$$
\begin{aligned}
& \hat{\delta} A_{1}=A_{\bar{z}}-\partial_{\bar{z}} W W^{-1}-W A_{\bar{z}} W^{-1}, \\
& \hat{\delta} A_{k}=\partial_{\lambda} A_{k}+(k-1) \lambda^{k-2}\left(\partial_{\bar{y}} W W^{-1}+W A_{\bar{y}} W^{-1}\right), \quad k \geqq 2,
\end{aligned}
$$

are "generalized" symmetries in the sense that they satisfy linearized equations of the SDYM hierarchy but, in addition to a polynomial part in $\lambda$, contain an "infinite tail" of negative powers of $\lambda$. The latter statement is obvious whereas the former one follows from the representation

$$
\hat{\delta} A_{k}=D_{k} J-\left[A_{k}, J\right], \quad k \geqq 1,
$$

and the definition of $\hat{\delta} A_{k}$ and $J$. By Theorem 3 the family $I_{k}=\left(I, \hat{\delta} A_{k}\right)$ gives another set of SDYM conservation laws.

Remark 14. Assuming the conditions of Remark 2 (i.e. $G=G L(n)$ and $A_{\bar{y}}, A_{\bar{z}} \in h$ ), one can further specialize the construction of conservation laws given in Theorem 3. Namely, using the representation (14):

$$
W=(\mathbf{1}+M) \exp Z
$$


where $\left.M \in \hat{n}\right|_{-}$and $\left.Z \in \hat{h}\right|_{-}$(see Remark 2), we conclude that the family $I_{k}=\left(K, D_{k} \partial_{\lambda} Z\right)$ provides additional conservation laws. Indeed, introduce another function

$$
J=W \partial_{\lambda} Z W^{-1}=(\mathbf{1}+M) \partial_{\lambda} Z(\mathbf{1}+M)^{-1}
$$

in (25) and use the intertwiner property of $W$ (see Remark 7) to obtain

$$
\hat{\delta} A_{k}=(\mathbf{1}+M) D_{k} \partial_{\lambda} Z(\mathbf{1}+M)^{-1} .
$$

Now using Theorem 3 with $K$ belonging to a Cartan subgroup of $G$ and the invariance property of $($,$) we get$

$$
I_{k}=\left(I, \hat{\delta} A_{k}\right)=\left(W K W^{-1}, W D_{k} \partial_{\lambda} Z W^{-1}\right)=\left(K, D_{k} \partial_{\lambda} Z\right) .
$$

In the next two sections we will demonstrate how these "universal" formulas yield all known results on hierarchies, conservation laws and symmetries for the integrable equations in $1+1$ and $2+1$ dimensions.

\section{Reductions to $1+1$ Dimensions}

In this section we discuss three well known $1+1$ dimensional reductions of the SDYM equations: the $N$-wave system, the nonlinear Schrödinger (NLS) equation and the Korteweg-de Vries (KdV) equation (see [15]). We will demonstrate how the appropriate reductions of the general scheme developed in the previous section yield these equations, their hierarchies, symmetries and conservation laws.

4.1. The $N$-Wave Reduction. Consider the SDYM equations with the gauge group $G=G L(n)$ and assume that the Yang-Mills potentials $A_{\mu}(\mathbf{x})$ do not depend on the variables $\bar{y}$ and $\bar{z}$ (in the complexified version of the SDYM equations). Denoting the remaining variables $y$ and $z$ by $x$ and $t$ respectively, so that $D_{1}=\partial_{x}$ and $D_{2}=\partial_{t}$, and choosing the gauge where $A_{\bar{z}}=A, A_{\bar{y}}=-B \in h$ (i.e. are diagonal matrices) and $A_{y}=U, A_{z}=V \in n$ (i.e. are off diagonal matrices), we can rewrite the SDYM linear system (4)-(5) as

$$
\begin{aligned}
& \partial_{x} \Psi=(U+\lambda A) \Psi, \\
& \partial_{t} \Psi=(V+\lambda B) \Psi .
\end{aligned}
$$

The reduced SDYM system, i.e. the compatibility condition for (26)-(27), reads

$$
\partial_{t} U-\partial_{x} V+[U, V]=0, \quad[A, V]=[B, U] .
$$

Assuming that ad $A$, ad $B$ are non-degenerate linear operators (while acting on off diagonal matrices), the second equation above can be easily solved: $U=[A, Q]$, $V=[B, Q], Q \in n$, and the resulting nonlinear system of PDE's:

$$
\left[A, \partial_{t} Q\right]-\left[B, \partial_{x} Q\right]+[[A, Q],[B, Q]]=0
$$

is called the $N$-wave system. Here $N=n(n-1)$ is the number of "elementary waves." Physically interesting cases require additional involutions of (26)-(27), and reduce the number of independent "waves" to $N=n(n-1) / 2$ (for further details see, e.g., $[12,16-18])$.

This reduction of the SDYM system to the $N$-wave system is quite natural and was presented in [19]. In addition to that, all general constructions for the SDYM system from Sect. 3 admit a natural reduction to this case. 


\section{Theorem 4.}

i) The reduced SDYM intertwiner $W(x, \lambda)$ has a representation

$$
W=(\mathbf{1}+M) \exp Z \text {, }
$$

where

$$
M(x, \lambda)=\left.\sum_{k=1}^{\infty} \frac{M_{k}(x)}{\lambda^{k}} \in \hat{n}\right|_{-}, \quad Z(x, \lambda)=\left.\sum_{k=1}^{\infty} \frac{Z_{k}(x)}{\lambda^{k}} \in \hat{h}\right|_{-},
$$

and $M_{k}(x)$ are local in $x$, i.e. are polynomials of matrix elements of $Q(x)$ and its derivatives.

ii) The SDYM hierarchy reduces to the $N$-wave hierarchy given by the following evolution equations, which are local in $x$ :

$$
\partial_{t_{k}} U=\partial_{x} \Phi_{k-1}-\left[U, \Phi_{k-1}\right], \quad t_{2}=t, \quad k \geqq 2,
$$

where $\Phi_{k}(x)$ are defined by

$$
\Phi=W B W^{-1}=(1+M) B(1+M)^{-1}=B+\sum_{k=1}^{\infty} \frac{\Phi_{k}(x)}{\lambda^{k}}
$$

and are local.

iii) The common wave function $\Psi$ for the $\mathrm{N}$-wave hierarchy is the reduction of that for the SDYM hierarchy and satisfies the system

$$
\partial_{t_{k}} \Psi=A_{k} \Psi, \quad k \geqq 1,
$$

where $t_{1}=x, A_{1}=U+\lambda A$, and

$$
A_{k}=\left(\lambda^{k-1} \Phi\right)_{+}=\sum_{l=0}^{k-1} \Phi_{k-l-1} \lambda^{l}, \quad k \geqq 2 .
$$

iv) Higher $N$-wave flows commute:

$$
\partial_{t_{k}} A_{l}-\partial_{t_{l}} A_{k}+\left[A_{l}, A_{k}\right]=0 .
$$

v) The formal recursion operator $R$ for the SDYM hierarchy reduces to the $N$-wave recursion operator $\Lambda$ such that

$$
\Phi_{k}=\Lambda^{k}(B), \quad k \geqq 1 .
$$

Proof. Statement i) follows from the recurrence relation (17) (see Remark 2), which in this case has the form

$$
\left[A, M_{k+1}\right]=\partial_{x} M_{k}+\sum_{l=1}^{k-1} M_{l}\left(U M_{k-l}\right)_{c}-\left(U M_{k}\right)_{n}, \quad\left[A, M_{1}\right]=-U,
$$

and obviously admits a unique solution local in $x$. Equation (16) in this case reads

$$
\partial_{x} Z=(U M)_{c}
$$

and determines $Z(x, \lambda)$. Statements ii)-iv) now follow from i) and general results in Sect. 3. In order to prove v), consider the reduction of recurrence relation (19):

$$
\operatorname{ad} A\left(\Phi_{k+1}\right)=\left(\partial_{x}-\operatorname{ad} U\right)\left(\Phi_{k}\right), \quad k \geqq 1,
$$

where $\Phi_{1}=B$. Formally $\Phi_{k+1}=R\left(\Phi_{k}\right)$, where $R=(\operatorname{ad} A)^{-1}\left(\partial_{x}-\operatorname{ad} U\right)$. However, in our particular case it is possible to "properly invert" the operator ad $A$. 
Namely, consider (28) and separate its Cartan and nilpotent components; one immediately concludes that for determining the Cartan part of $\Phi_{k+1}$ the operator ad $A$ can be properly inverted whereas for determining the Cartan part of $\Phi_{k+1}$ one needs to apply the operator $\partial_{x}^{-1}$ ad $U$ to its nilpotent part. This results in the formula $\Phi_{k+1}=\Lambda\left(\Phi_{k}\right)$ with the integro-differential operator $\Lambda$. However, since all $\Phi_{k}$ are local, quantities ad $U\left(\left(\Phi_{k}\right)_{n}\right)$ are total derivatives in $x$ so that $\partial_{x}$ can be canonically inverted.

Remark 15. The construction of the SDYM connections from the members of the hierarchy, as shown in Remark 10, can be carried out here and in other $1+1$ reductions as well. Thus one obtains nontrivial self-dual connections in $\mathbb{C}^{4}$ out of solutions of $1+1$ soliton hierarchies (cf. [20]).

In addition, we have

\section{Theorem 5.}

i) The family of symmetries $\delta^{(k)} A_{l}$ associated with the generating function $J^{(k)}=\left(\lambda^{k} \Phi\right)_{-}$reduces to $\delta^{(k)} A_{l}=-\partial_{t_{k}} A_{l}$, where the starting symmetries $\delta^{(k)} A_{1}=\delta^{(k)} U$ are given by

$$
\delta^{(k)} U=\operatorname{ad} A \Lambda^{k-1}(\operatorname{ad} A)^{-1}\left(\delta^{(1)} U\right), \quad \delta^{(1)} U=[U, B] .
$$

ii) The $N$-wave hierarchy can be written as

$$
\partial_{t_{k}} U=-\operatorname{ad} A \Lambda^{k-1}(\operatorname{ad} A)^{-1}\left(\delta^{(1)} U\right) .
$$

iii) Conservation laws associated with the symmetries $\delta^{(k)} A_{1}$ become trivial under the $N$-wave reduction, i.e. they are total derivatives of functions local in $x$. The family of nontrivial local conservation laws is given by a reduction of the family constructed in Remark 14, is parametrized by element $K$ from a Cartan subgroup of $G$ and has the generating function

$$
I_{1}(\lambda)=\partial_{x}\left(K, \partial_{\lambda} Z\right)
$$

Proof. Starting from the definition of the symmetries $\delta^{(k)} A_{l}$ (see Theorem 2), the following calculation, which uses commutativity of higher flows and the differential equation for $\Phi(22)$ :

$$
\begin{aligned}
\delta^{(k)} A_{l} & =\partial_{t_{l}}\left(\lambda^{k-1} \Phi\right)_{-}+\left[\left(\lambda^{k-1} \Phi\right)_{-}, A_{l}\right] \\
& =\partial_{t_{l}}\left(\lambda^{k-1} \Phi-A_{k}\right)+\left[\lambda^{k-1} \Phi-A_{k}, A_{l}\right] \\
& =\lambda^{k-1}\left(\partial_{t_{l}} \Phi-\left[A_{l}, \Phi\right]\right)-\partial_{t_{l}} A_{k}+\left[A_{l}, A_{k}\right] \\
& =-\partial_{t_{k}} A_{l},
\end{aligned}
$$

proves i). Statement ii) follows from the calculation (see Theorem 2, Remark 12 and Eq. (19)):

$$
\delta^{(k)} U=\delta^{(k)} A_{1}=-\left[A, \Phi_{k}\right]=\operatorname{ad} A \Lambda^{k-1}(\operatorname{ad} A)^{-1}\left(\delta^{(1)} U\right),
$$

since $\delta^{(1)} U=-\left[A, \Phi_{1}\right]$.

To prove iii), consider the definition of conservation laws $I_{1}^{(k)}=\left(I, \delta^{(k)} A_{1}\right)$, where $($,$) is the invariant bilinear form and the differential equation \partial_{x} I=\left[A_{1}, I\right]$. Writing

$$
\delta^{(k)} A_{1}=-\partial_{t_{k}} A_{1}=-\partial_{x} A_{k}+\left[A_{1}, A_{k}\right],
$$


then yields

$$
I_{1}^{(k)}=-\partial_{x}\left(I, A_{k}\right)
$$

Since $I=W K W^{-1}$ and $W$ is local in $x$ it shows the triviality of the conservation laws $I_{1}^{(k)}$. To obtain the nontrivial ones use the construction from Remark 14, which yields the generating function

$$
I_{1}(\lambda)=\partial_{x}\left(K, \partial_{\lambda} Z\right)
$$

for the family of conservation laws parametrized by Cartan element $K$. Although they are total derivatives they are nontrivial due to the differential equation (16)

$$
\partial_{x} Z=(U M)_{c} .
$$

$Z$ and $\partial_{\lambda} Z$ are non-local in $x$ so that densities in expansion of $I_{1}(\lambda)$ produce nontrivial integrals of motion (integrals over the period for the periodic case or over $\mathbb{R}$ in the rapidly decreasing case).

Remark 16. It is instructive to note that $N$-wave hierarchies parametrized by different elements $B \in h$ commute; the "universal" proof of Theorem 1 applies verbatim to this case. This shows that there is a $N-1$-"infinite" family of different higher $N$-wave "times" according to the dimension of $h$ (the unit element in $h$ gives trivial flows). In particular, the hierarchy which corresponds to the choice $B=A$ starts with the linear equation

$$
\partial_{t} U=\partial_{x} U
$$

which describes the translation invariance of the $N$-wave system.

\subsection{NLS Reduction. The NLS equation}

$$
\partial_{t} \psi=-\partial_{x}^{2} \psi \pm 2|\psi|^{2} \psi
$$

can be obtained as a second member of the 2-wave hierarchy with appropriate choice of $A, B, U$ and $V$ and, therefore, may be considered as a reduction of the SDYM hierarchy (with higher NLS flows obtained from higher SDYM flows). However, there is also a way of getting NLS directly from SDYM system. Namely, consider the following SDYM reduction [15]: the gauge group $G$ is $S L(2)$ and Yang-Mills potentials are independent of the variables $y-\bar{y}$ and $\bar{z}$. Denoting the remaining variables $y$ and $z$ as $x$ and $t$, so that $D_{1}=\partial_{x}, D_{2}=\partial_{t}-\lambda \partial_{x}$, and choosing the gauge $A_{\bar{y}}=0, A_{\bar{z}}=H \in h, A_{y}=U \in n, A_{z}=V \in g$, the SDYM linear system (4)-(5) takes the form

$$
\partial_{x} \Psi=(U+\lambda H) \Psi, \quad\left(\partial_{t}-\lambda \partial_{x}\right) \Psi=V \Psi,
$$

and its compatibility reads

$$
\partial_{t} U-\partial_{x} V+[U, V]=0, \partial_{x} U=[H, V] .
$$

Using the second equation and the Cartan component of the first, one can express $V$ in terms of $U$ and its $x$ derivatives (the result will be local in $x$ ); the nilpotent part of the first equation in the compatibility condition yields the nonlinear evolution equation for $U$. When

$$
H=-i\left(\begin{array}{rr}
1 & 0 \\
0 & -1
\end{array}\right), \quad U=\left(\begin{array}{rr}
0 & \psi \\
\mp \bar{\psi} & 0
\end{array}\right),
$$


so that

$$
V=i\left(\begin{array}{cc}
|\psi|^{2} & \partial_{x} \psi \\
\pm \partial_{x} \bar{\psi} & -|\psi|^{2}
\end{array}\right)
$$

the compatibility condition yields the NLS equation.

If one replaces $x$-derivatives in the second equation in the linear system by the first equation the result will be the standard linear system for NLS equation (see, e.g., $[12,18,21])$. All constructions of Sect. 3 go verbatim into those for the NLS case: hierarchy, symmetries and conservation laws can be derived immediately. We omit the derivation, since it repeats that for the $N$-wave system.

4.3. KdV Reduction. The $\mathrm{KdV}$ reduction is obtained by imposing the same coordinate dependence and the choice of gauge group as for the NLS reduction. Namely, denote by $E_{+}$and $E_{-}$the $s l(2)$ roots, and choose the Yang-Mills potentials to be

$$
\begin{gathered}
A_{\bar{z}}=E_{-}=\left(\begin{array}{ll}
0 & 0 \\
1 & 0
\end{array}\right) \in n_{-}, \quad A_{y}=U=u E_{-}+E_{+}=\left(\begin{array}{ll}
0 & 1 \\
u & 0
\end{array}\right) \in \hat{n}, \\
A_{z}=V \in \hat{g}, \quad A_{\bar{y}}=E \in \hat{n},
\end{gathered}
$$

where $u(x, t)$ is the $\mathrm{KdV}$ field. The SDYM linear system (4)-(5) takes the form

$$
\partial_{x} \Psi=\left(U+\lambda E_{-}\right) \Psi, \quad\left(\partial_{t}-\lambda \partial_{x}\right) \Psi=(V-\lambda E) \Psi,
$$

whose compatibility reads

$$
\partial_{t} U-\partial_{x} V+[U, V]=0, \partial_{x} U-\partial_{x} E=[E, U]+\left[E_{-}, V\right] .
$$

Using the second equation in (29) and Cartan and $n_{+}$components of the first one can express $V$ and $E$ in terms of $u$ and its $x$-derivatives:

$$
V=\frac{1}{4}\left(\begin{array}{cc}
\partial_{x} u & -2 u \\
-2 u^{2}+\partial_{x}^{2} u & -\partial_{x} u
\end{array}\right), \quad E=\frac{u}{2} E_{-},
$$

so that the remaining $n_{-}$component of the first equation yields the $\mathrm{KdV}$ equation:

$$
\partial_{t} u=\frac{1}{4}\left(\partial_{x}^{3} u-6 u \partial_{x} u\right) .
$$

Remark 17. Notice that the gauge choice for the $\mathrm{KdV}$ reduction is different from that in Lemma 1: although $\left[A_{\bar{y}}, A_{\bar{z}}\right]=0$, the gauge potential $A_{\bar{y}}$ is not constant. However, according to Remark 11, this gauge choice differs from that in Lemma 1 by a gauge transformation $A \mapsto A f^{-1}$ with $f=1+\frac{1}{2} \partial_{x}^{-1} u E_{-}$. In this gauge $A_{\bar{y}} f^{-1}=0, A_{\bar{z}} f^{-1}=E_{-}$.

Remark 18. Choosing the gauge from the previous remark, we can use the general construction from Sect. 3 based on the intertwiner $W$. However, in Lemma 2 the "bare" SDYM solutions were chosen to be $A_{y}=A_{z}=0$ so that the "bare" $A_{1}=A_{\bar{z}}$. In $\mathrm{KdV}$ case, due to the special choice of the potential $A_{y}=U$, natural "bare" solution is $A_{z}=0, A_{y}=E_{+}$so that one needs to use $\Lambda=E_{+}+\lambda E_{-}$as a "bare" $A_{1}$ in Lemma 2. These peculiarities show that $\mathrm{KdV}$ equation is a rather special reduction of the general formalism of the IST method contrary to the $N$-wave and NLS cases, which are generic in their classes (cf. the discussion in [12]). 
These special features of the $\mathrm{KdV}$ reduction result in the following form of the intertwiner

$$
W(x, \lambda)=W_{0}+\sum_{n=1}^{\infty} \frac{W_{n}(x)}{\lambda^{n}},
$$

where

$$
W_{0}=f=1+\frac{1}{2} \partial_{x}^{-1} u E_{-},
$$

(cf. Remarks 11 and 18), which "dresses" the "bare" SDYM hierarchy $A_{1}=\Lambda$, $A_{k}=0, k \geqq 2$. It satisfies the equations

$$
\hat{W}\left(\partial_{x}-\Lambda\right) \hat{W}^{-1}=\partial_{x}-A_{1}=\partial_{x}-U_{0}-\Lambda, \quad U_{0}=U-E_{+},
$$

and

$$
\hat{W}\left(\partial_{t_{k}}-\lambda^{k-1} \partial_{x}\right) \hat{W}^{-1}=\partial_{t_{k}}-\lambda^{k-1} \partial_{x}-A_{k}
$$

where

$$
A_{k}=\partial_{t_{k}} f f^{-1}+\left(\lambda^{k-1} \Phi\right)_{+}, \quad k \geqq 2, \quad \Phi=-\partial_{x} W W^{-1},
$$

which determine the $\mathrm{KdV}$ hierarchy. The coefficients $W_{n}$ are determined via $\partial_{x} W=A_{1} W-W \Lambda$, which is obtained from (30). Replacing the $x$-derivatives in evolution equations with the help of the first equation in the KdV linear system, we can rewrite the KdV hierarchy as

$$
\partial_{x} \Psi=A_{1} \Psi, \quad \partial_{t_{k}} \Psi=\tilde{A}_{k} \Psi, \quad k \geqq 2,
$$

where

$$
\tilde{A}_{k}=\partial_{t_{k}} f f^{-1}+\left(\lambda^{k-1} \Phi\right)_{+}+\lambda^{k-1} A_{1}=\left(\lambda^{k-1} \tilde{\Phi}\right)_{+}+\partial_{t_{k}} f f^{-1}
$$

and

$$
\tilde{\Phi}=W \Lambda W^{-1}=\sum_{n=-1}^{\infty} \frac{\tilde{\Phi}_{n}}{\lambda^{n}}, \quad \tilde{\Phi}_{-1}=E_{-} .
$$

Remark 19. Setting in the previous formula $k=1$ we get

$$
\tilde{A}_{1}=A_{1}-E+\partial_{t_{1}} f f^{-1}
$$

which results in the equation

$$
\partial_{t_{1}} u=\partial_{x} u
$$

- the trivial "starting" member of the $\mathrm{KdV}$ hierarchy.

Remark 20. Defining $A_{1}=B_{1}^{f}, \tilde{A}_{k}=B_{k}^{f}, k \geqq 2$, and $\tilde{\Phi}=\Omega^{f}=f \Omega f^{-1}-$ a gauge transformation by $f$ (cf. Remark 11 ) - we obtain for the new potentials $B_{k}$

$$
B_{k}=\left(\lambda^{k-1} \Omega\right)_{+}, \quad k \geqq 1,
$$

Since $\Lambda^{2}=\lambda \mathbf{1}$, we can rewrite the last formula as

$$
B_{k}=\left(\Omega^{2 k-1}\right)_{+},
$$

which shows that KdV hierarchy contains only "odd" flows - "even" ones are given by the potentials $\Omega^{2 k}=\lambda^{k} 1$ and result in trivial (static) equations. 
Contrary to the coefficients $W_{n}$, the coefficients $\tilde{\Phi}_{n}$ are local in $x$ and can be obtained from the recurrence relation

$$
\left[E_{-}, \tilde{\Phi}_{n+1}\right]=\partial_{x} \tilde{\Phi}_{n}-\left[U, \tilde{\Phi}_{n}\right],
$$

which follows from the differential equation

$$
\partial_{x} \tilde{\Phi}=\left[A_{1}, \tilde{\Phi}\right] .
$$

In terms of these coefficients the higher $\mathrm{KdV}$ flows have the form

$$
\partial_{t_{k}} U=\partial_{x} V_{k}-\left[U, V_{k}\right], \quad k \geqq 2,
$$

where $V_{k}=\tilde{\Phi}_{k-1}+\partial_{t_{k}} f f^{-1}$.

Denoting by $\tilde{\Phi}^{c}, \widetilde{\Phi}^{ \pm}$the Cartan and $n_{ \pm}$components of $\tilde{\Phi}$, one can easily recover from (32) the well known recursion operator for the KdV hierarchy, which can be considered as a specialization of the formal expression $R=\left(\operatorname{ad} E_{-}\right)^{-1}\left(\partial_{x}-\operatorname{ad} U\right)$. Namely, using Eq. (32) we get

$$
\partial_{t_{k}} u=4 \tilde{\Phi}_{k}^{c}=4 \mathbf{I}\left(\tilde{\Phi}_{k-1}^{c}\right)=\ldots=4 \mathbf{I}^{k-1}\left(\tilde{\Phi}_{1}^{c}\right), \quad \tilde{\Phi}_{1}^{c}=\frac{1}{4} \partial_{x} u
$$

where the integro-differential operator $\mathbf{I}$,

$$
\mathbf{I}=\left(\frac{1}{4} \partial_{x}^{3}-u \partial_{x}-\frac{1}{2} \partial_{x} u\right) \partial_{x}^{-1}
$$

is the well known recursion operator for the $\mathrm{KdV}$ hierarchy (see $[25,26]$ ).

Remark 21. It is instructive to compare this approach with the standard one based on the Schrödinger equation. Namely, the first order linear system

$$
\partial_{x} \Psi=\left(U+\lambda E_{-}\right) \Psi
$$

is nothing but the canonical Frobenius form of the second order differential equation

$$
-\partial_{x}^{2} f+u(x) f=-\lambda f
$$

(Schrödinger eigen-value problem with the energy $E=-\lambda$ ). Denoting by $f_{1}$ and $f_{2}$ the two linearly independent solutions of the Schrödinger equation, we have explicitly

$$
\Psi=\left(\begin{array}{cc}
f_{1} & f_{2} \\
\partial_{x} f_{1} & \partial_{x} f_{2}
\end{array}\right)
$$

so that by choosing $f_{1}=\exp (k x) \phi_{1}(x, k), f_{2}=f_{1}(x,-k)=\exp (-k x) \phi_{2}(x, k)$, where $\phi_{2}(x, k)=\phi_{1}(x,-k), k=\sqrt{\lambda}$, and $\phi_{1,2}$ are power series in $k^{-1}$, we get

$$
W=\frac{1}{2 k}\left(\begin{array}{cc}
k\left(\phi_{1}+\phi_{2}\right) & \phi_{1}-\phi_{2} \\
k \partial_{x}\left(\phi_{1}+\phi_{2}\right)+k^{2}\left(\phi_{1}-\phi_{2}\right) & \partial_{x}\left(\phi_{1}-\phi_{2}\right)+k\left(\phi_{1}+\phi_{2}\right)
\end{array}\right) .
$$

It follows from this representation that $W(x, \lambda)$ is even in $k$ and indeed admits expansion in integer negative powers of $\lambda=k^{2}$. Moreover, from this representation it can be shown that even if the coefficients $W_{n}$ are nonlocal in $x$, the coefficients $\tilde{\Phi}_{n}$ are local. (Recall the standard fact from the Schrödinger equation that logarithmic 
$x$-derivative of $\phi(x, k)$ admits expansion in $k^{-1}$ whose coefficients are local in $x$; expressions of this type enter in $\widetilde{\Phi}$.)

Using the previous remark we can relate our approach to $\mathrm{KdV}$ hierarchy, based on the general intertwiners for the SDYM system with the standard one by Gelfand-Dikii [22] and Adler [23]. Namely, using (32) we see that $\tilde{\Phi}_{k}^{c}=-\frac{1}{2} \partial_{x} \tilde{\Phi}_{k}^{+}$ so that the $\mathrm{KdV}$ hierarchy can be written as

$$
\partial_{t_{k}} u=-2 \partial_{x} \tilde{\Phi}_{k}^{+}
$$

where $\tilde{\Phi}_{k}^{+}$satisfies the recurrence relation

$$
\tilde{\Phi}_{k}^{+}=\hat{\mathbf{I}}\left(\tilde{\Phi}_{k-1}^{+}\right)
$$

with $\hat{\mathbf{I}}=\partial_{x}^{-1} \mathbf{I} \partial_{x}$. Moreover, it follows from Eq. (33) that the function $\tilde{\Phi}^{+}(x, \lambda)$ satisfies a third order linear equation

$$
\partial_{x}^{3} \tilde{\Phi}^{+}=2\left(\partial_{x} u\right) \tilde{\Phi}^{+}+4(u+\lambda) \partial_{x} \tilde{\Phi}^{+},
$$

which is the well known equation for the restriction of the resolvent kernel of the Schrödinger operator $L=\partial_{x}^{2}-u$ on the diagonal (actually this equation goes back to Hermite [24]; see also [22]). One of the main results of Gelfand-Dikii's algebraic analysis of fractional powers of the Schrödinger operator is that

$$
\left[L,\left(L^{k-\frac{1}{2}}\right)_{+}\right]=-2 \partial_{x} \tilde{\Phi}_{k}^{+},
$$

where here + denotes the differential part of the formal fractional power of $L$. Therefore we can rewrite $\mathrm{KdV}$ hierarchy as

$$
\partial_{t_{k}} L=\left[L,\left(L^{k-\frac{1}{2}}\right)_{+}\right] \text {, }
$$

which is a key formula from Adler's approach.

Consequently the framework we have exhibited for the SDYM symmetries and conservation laws contains those for the $\mathrm{KdV}$, which are well known. For example, the conservation laws for the KdV hierarchy can be obtained from the SDYM ones by using Remark 13 and are given by

$$
\partial_{t_{k}}\left(\tilde{\Phi}, E_{-}\right)=\partial_{x}\left(\tilde{\Phi}, \frac{d \tilde{A}_{k}}{d \lambda}\right), \quad k \geqq 2,
$$

where (, ) stands for the Cartan-Killing form for $g l(2)$. The same applies to the symmetries and we will not repeat arguments presented in $N$-wave and NLS cases.

These examples illustrate an essential point of this paper; namely integrable $1+1$ systems are obtained from SDYM as reductions. Similarly, the RiemannHilbert factorization problem for SDYM system reduces to all these cases as well yielding the standard Riemann-Hilbert problem of the IST formalism for the $1+1$ systems.

\section{Reductions to $2+1$ Dimensions}

The basic examples considered in the previous section admit a generalization to the case of two spatial and one time variables and it is instructive to see how these multidimensional systems can be obtained as SDYM reductions. It is well known that the spatial variables in $2+1$ integrable systems are treated differently and this difference is central in the IST method [27]; (see also [28, 29]). These features are 
also manifest in the SDYM reductions and to the best of our knowledge there is no natural way to obtain these systems as SDYM reductions with a finite dimensional gauge group (the second spatial variable does not come naturally from the SDYM space-time variables).

Here we outline an approach which allows us to obtain multidimensional systems as SDYM reductions for a special choice of an infinite dimensional gauge group. Namely, we assume that the gauge Lie algebra $g$ is the infinite dimensional Lie algebra of all formal matrix pseudodifferential operators in auxiliary variable $s$ :

$$
g=\left\{\sum_{l=-\infty}^{l \ll \infty} a_{l} \partial_{s}^{l}\right\},
$$

where $a_{l}$ belong to a ring of $n \times n$ matrix-functions of $s$. Clearly $g$ is an associative algebra with a multiplication given by a standard composition of pseudodifferential operators with matrix coefficients, so that $g$ has a natural Lie algebra structure. As in Sect. 3, we define $\hat{g}$ to be a Lie algebra of $g$-valued functions on $\mathbb{R}^{4}$ involving the "primitives" with respect to the certain first order differential operator with constant coefficients: $\partial_{\bar{z}}-A \partial_{s}$ (see below). One can pass from a formal to the rigorous theory provided one specifies appropriate functional classes. The Lie algebra $\hat{g}$ admits the decomposition

$$
\hat{g}=\hat{g}_{-} \oplus \hat{g}_{+},
$$

where the subalgebras $\hat{g}_{-}$and $\hat{g}_{+}$consist, respectively, of power series in $\partial_{s}^{-1}$ without a constant term, and of polynomials in $\partial_{s}$.

Now our claim is that it is a SDYM system with such a choice of gauge group that realizes reductions to $2+1$ integrable systems, where the second spatial variable is obtained from the "fiber" variable $s$ !

More precisely, we choose the gauge as $A_{\bar{y}}=A_{\bar{z}}=0$ and assume that two remaining Yang-Mills potentials $A_{y}, A_{z} \in \hat{g}_{+}$are first order in $\partial_{s}$, i.e.

$$
A_{y}=U+A \partial_{s}, \quad A_{z}=V+B \partial_{s},
$$

where $A$ and $B$ are commuting matrices independent of $\mathbf{x}$ and $s$. In this special gauge the linear system (4)-(5) takes the form

$$
\begin{aligned}
& \left(\partial_{y}+\lambda \partial_{\bar{z}}\right) \Psi=\left(U+A \partial_{s}\right) \Psi, \\
& \left(\partial_{z}-\lambda \partial_{\bar{y}}\right) \Psi=\left(V+B \partial_{s}\right) \Psi .
\end{aligned}
$$

The equation, $F_{y \bar{y}}+F_{z \bar{z}}=0$, which arises as the coefficient of $\lambda$ in the compatibility condition of (34)-(35), can be satisfied by setting $U=\partial_{\bar{z}} C, V=-\partial_{\bar{y}} C$ for certain $C \in \hat{g}$. A special class of solutions for the remaining SDYM equation, $F_{y z}=0$, can be now obtained by considering a solution of the linear system (34)-(35) corresponding to a particular choice of the spectral parameter $\lambda$, e.g. $\lambda=1$. In this case concrete representations for the solution $\Psi$ can be obtained by the IST technique. It is worth noting that the "fiber" variable $s$ plays a crucial role even within the framework of solving the remaining equation $F_{y z}=0$ as the various coefficients of the $F_{y z} \in \hat{g}_{+}$produce a system of nonlinear PDE's. Keeping these remarks in mind we will consider the linear system (34)-(35) for $\lambda=1$ :

$$
\begin{aligned}
& \left(\partial_{y}+\partial_{\bar{z}}\right) \Psi=\left(U+A \partial_{s}\right) \Psi \\
& \left(\partial_{z}-\partial_{\bar{y}}\right) \Psi=\left(V+B \partial_{s}\right) \Psi .
\end{aligned}
$$


All general constructions in Sect. 3 can then be adapted to this case. Namely, now the intertwiner $W \in \hat{g}$ has the form

$$
W=\mathbf{1}+\sum_{k=1}^{\infty} W_{k} \partial_{y}^{-k}
$$

and satisfies the equation

$$
D_{1}-A_{1}=\hat{W}\left(D_{1}-A \partial_{s}\right) \hat{W}^{-1},
$$

or

$$
D_{1} W+W A \partial_{s}=A_{1} W,
$$

where $D_{1}=\partial_{y}+\partial_{\bar{z}}$ and "bare" $A_{1}$ is $A \partial_{s}$. The SDYM hierarchy can be introduced by dressing the "bare" Yang-Mills potentials $B \partial_{s}^{k-1}$ :

$$
D_{k}-A_{k}=\hat{W}\left(D_{k}-B \partial_{s}^{k-1}\right) \hat{W}^{-1},
$$

where $D_{k}=\partial_{z_{k}}-\partial_{\bar{y}}$, so that

$$
D_{k} \Psi=A_{k} \Psi, \quad k \geqq 2,
$$

where

$$
A_{k}=\partial_{t_{k}} W W^{-1}+\Phi^{(k)}=\left.\Phi^{(k)}\right|_{+}
$$

(cf. Remark 7) and

$$
\Phi^{(k)}=W B \partial_{s}^{k-1} W^{-1}=\sum_{l=0}^{\infty} \Phi_{l}^{(k)} \partial_{s}^{k-l-1}, \quad \Phi_{0}^{(k)}=B .
$$

From (39) one finds that $\Phi^{(k)}$ satisfies the differential equation

$$
D_{1} \Phi^{(k)}=\left[U+A \partial_{s}, \Phi^{(k)}\right] .
$$

Remark 22. Since $\hat{W}$ acts on the elements of $\hat{g}$, which are the (formal) pseudodifferential operators in the vriable $s$, using terminology from [30] we can call it an operand (operator acting on operators).

Remark 23. For $k=2$ we get from (42) and (44) that the second Yang-Mills potential has the form $A_{2}=V+B \partial_{s}$, thus recovering the original SDYM system with this infinite dimensional algebra $g$.

Remark 24. Although $\Phi^{(k)}$ satisfy the same differential equation (44) they are different elements in $\hat{g}$ (since $\partial_{s}$ is now a derivation in $\hat{g}$ ) and all of them are needed for the construction of the hierarchy (cf. the usual case in Sect. 3 where, because $\lambda$ was the central element in $\hat{g}$, a single function $\Phi$ generated the entire hierarchy).

Other constructions in Sect. 3 can be adapted in a similar manner; we only mention that if $A$ and $B$ are Cartan elements in $g l(n)$, then the intertwiner $W$ admits a representation similar to that of Remark 2:

$$
W=(\mathbf{1}+M) \exp Z
$$

with appropriate $M$ and $Z$.

5.1. The $N$-Wave Reduction. The main idea for $2+1$ SDYM reductions is to consider SDYM potentials depending only on two spatial variables $y$ and $z$, which 
will be denoted by $x$ and $t$ respectively; the "fiber" variable $s$, denoted as $y$, will provide the second spatial variable for $2+1$ systems. What is important is that in this reduction there is no spectral parameter $\lambda$ anymore so that the linear system we get from (36)-(37) reads

$$
\begin{aligned}
& \partial_{x} \Psi=\left(U+A \partial_{y}\right) \Psi, \\
& \partial_{t} \Psi=\left(V+B \partial_{y}\right) \Psi,
\end{aligned}
$$

which is the well known linear system associated with the $N$-wave equations (see, e.g., [27]).

Remark 25. The reduced SDYM system is equivalent to the compatibility condition of the system (46)-(47), which is a single "zero curvature" equation. Nevertheless, due to the infinite dimensional fiber $g$, this system is meaningful and the examples presented clearly illustrate this.

Choosing the coefficients in the linear system (46)-(47) in the same way as for the $1+1$ case (with additional dependence on $y$ !) we get the compatibility condition

$$
\partial_{t} U-\partial_{x} V+[U, V]-B \partial_{y} U+A \partial_{y} V=0, \quad[A, V]=[B, U]
$$

which results in the nonlinear PDE

$$
\left[A, \partial_{t} Q\right]-\left[B, \partial_{x} Q\right]-B\left[A, \partial_{y} Q\right]+A\left[B, \partial_{y} Q\right]+[[A, Q],[B, Q]]=0,
$$

where $U=[A, Q], V=[B, Q]$.

Remark 26. The linear system (46)-(47) can be obtained from that for the $1+1$ case - (26)-(27) - by formally replacing the spectral parameter $\lambda$ by $\partial_{y}$. This is a standard procedure for deriving the $2+1$ integrable versions of the $1+1$ systems. However, in the full SDYM case one can not simply replace $\lambda$ by $\partial_{s}$, because then the differential operators $D_{1}$ and $D_{2}$ being second order would not satisfy the derivation property (Leibniz rule) which was essential for our constructions in Sect. 3. One can formally use this procedure only after reduction, when $D_{1}$ and $\mathrm{D}_{2}$ would still be of the first order.

Proceeding in the same manner as in the previous section one can derive all known features of the $N$-wave hierarchy in $2+1$ case. In particular, using this approach it is especially clear why higher $N$-wave equations are nonlocal in $x$ and $y$. Namely, in the expansion (45) of the intertwiner $W$ the coefficients $M_{k}$ will be local in $x$ and $y$ whereas the coefficients $Z_{k}$ will be nonlocal since one needs to invert the first order differential operator with constant coefficients $\partial_{x}+A \partial_{y}$. In the $1+1$ case $\Phi=W B W^{-1}=(\mathbf{1}+M) B(\mathbf{1}+M)^{-1}$ and has coefficients $\Phi_{k}$ which are local in $x$, whereas in this case, since $\partial_{y}^{k-1}$ does not commute with $\exp Z$, we have

$$
\Phi^{(k)}=(\mathbf{1}+M) \exp Z B \partial_{y}^{k-1} \exp (-Z)(\mathbf{1}+M)^{-1} .
$$

This formula shows that nonlocal terms are present in $\Phi_{l}^{(k)}$ as well as in the higher flows. However for the $N$-wave case the coefficient $A_{2}=\left.\Phi^{(2)}\right|_{+}$is still local as expected since $A_{2}$ is one of the Yang-Mills potentials.

From Eqs. (43) and (44) one can write down the following recurrence relations for determining the coefficients $\Phi_{l}^{(k)}$ :

$$
\left[A, \Phi_{l+1}^{(k)}\right]=\partial_{x} \Phi_{l}^{(k)}-\left[U, \Phi_{l}^{(k)}\right]-A \partial_{y} \Phi_{l}^{(k)}+\sum_{p=1}^{l} C_{p}^{k, l} \Phi_{l-p}^{(k)} \partial_{y}^{p} U,
$$


where $C_{p}^{k, l}$ are certain binomial coefficients. From these it is possible to give a precise meaning to the formal recursion operator $R$ introduced in Sect. 3 consistent with the known results [31,32].

The evolution equations for $N$-wave hierarchy can be explicitly written as

$$
\begin{aligned}
\partial_{t_{k}} U & =\partial_{x} \Phi_{k-1}^{(k)}-\left[U, \Phi_{k-1}^{(k)}\right]-A \partial_{y} \Phi_{k-1}^{(k)}+\sum_{p=1}^{k-1} C_{p}^{k, k-1} \Phi_{k-1-p}^{(k)} \partial_{y}^{p} U \\
& =\left[A, \Phi_{k}^{(k)}\right],
\end{aligned}
$$

where the last equality follows from the recurrence relations for the $\Phi_{l}^{(k)}$. One can further pursue along these line obtaining symmetries, conservation laws, etc., from those for the SDYM case. We believe that previous examples demonstrated how to do it so we will omit these routine (though sometimes lengthy!) calculations.

5.2. The D-S Reduction. The D-S system is a natural $2+1$ generalization of the NLS equation and can be obtained as a second member in the 2-wave hierarchy. Namely, assume that

$$
A=H=\left(\begin{array}{rr}
1 & 0 \\
0 & -1
\end{array}\right), \quad U=\left(\begin{array}{ll}
0 & q \\
r & 0
\end{array}\right)
$$

and consider the linear system

$$
\begin{aligned}
\partial_{x} \Psi & =\left(U+H \partial_{y}\right) \Psi, \\
\partial_{t} \Psi=\left.\Phi^{(3)}\right|_{+} \Psi & =\left(V_{0}+V_{1} \partial_{y}+V_{2} \partial_{y}^{2}\right) \Psi,
\end{aligned}
$$

where $V_{1}=\kappa U$ and

$$
V_{2}=\frac{1}{2}\left(\begin{array}{cc}
2 R_{1} & \kappa\left(\partial_{x}+\partial_{y}\right) q \\
-\kappa\left(\partial_{x}-\partial_{y}\right) r & 2 R_{2}
\end{array}\right)
$$

with $R_{1}$ and $R_{2}$ satisfying the equations

$$
\begin{aligned}
& \left(\partial_{x}-\partial_{y}\right) R_{1}=-\frac{\kappa}{2}\left(\partial_{x}+\partial_{y}\right)(q r), \\
& \left(\partial_{x}+\partial_{y}\right) R_{2}=\frac{\kappa}{2}\left(\partial_{x}-\partial_{y}\right)(q r) .
\end{aligned}
$$

The compatibility condition results in the following system of PDE's for $q$ and $r$ :

$$
\begin{aligned}
& \partial_{t} q=\frac{\kappa}{2}\left(\partial_{x}^{2}+\partial_{y}^{2}\right) q+\left(R_{1}-R_{2}\right) q, \\
& \partial_{t} r=-\frac{\kappa}{2}\left(\partial_{x}^{2}+\partial_{y}^{2}\right) r+\left(R_{1}-R_{2}\right) r,
\end{aligned}
$$

where

$$
\left(\partial_{x}^{2}-\partial_{y}^{2}\right)\left(R_{1}-R_{2}\right)=-\kappa\left(\partial_{x}^{2}+\partial_{y}^{2}\right)(q r) .
$$

This nonlocal (after eliminating $R_{1}-R_{2}$ ) system of nonlinear PDE's for $q$ and $r$ is called the D-S system. The physically interesting cases correspond to the special reductions $q=\psi, r= \pm \bar{\psi}$ (after the rescaling $\partial_{y} \mapsto \sigma \partial_{y}$ and setting $\kappa=i / \sigma^{2}$, where $\sigma^{2}= \pm 1$. 
All general constructions in Sect. 3 are valid in the D-S case and the results concerning its hierarchy, conservation laws, symmetries and recursion operator, follow.

5.3. KP Reduction. The KP reduction is similar to that of $\mathrm{KdV}$ in the previous section with the only difference that now the "fiber" variable $s$, denoted by $y$, is the second spatial variable for the KP field $u(x, y, t)$. We can write down the KP linear system as

$$
\begin{aligned}
& \partial_{x} \Psi=\left(U+E-\partial_{y}\right) \Psi=A_{y} \Psi, \\
& \partial_{t} \Psi=\left(V-E \partial_{y}+A_{y} \partial_{y}\right) \Psi=A_{z} \Psi,
\end{aligned}
$$

where $U, E_{-}$and $E$ are the same as in Sect. 4.3 and $V$ has the form

$$
V=\frac{1}{4}\left(\begin{array}{cc}
\alpha+\partial_{x} u & -2 u \\
\partial_{x}^{2} u-2 u^{2}+\partial_{y} u & \alpha-\partial_{x} u
\end{array}\right)
$$

where $\alpha$ satisfies

$$
\partial_{x} \alpha=-3 \partial_{y} u \text {. }
$$

Eliminating $\alpha$ from the compatibility condition for the system (48)-(49) one gets the $\mathrm{KP}$ equation

$$
\partial_{t} u=\frac{1}{4}\left(\partial_{x}^{3} u-6 u \partial_{x} u+3 \partial_{x}^{-1}\left(\partial_{y}^{2} u\right)\right) .
$$

Using the general construction at the beginning of this section one can derive the KP hierarchy. In doing this one should keep in mind the special features of the $\mathrm{KP}$ gauge, which were already present in the KdV case (see Sect. 4.3). Namely, consider the bare potential in (48) to be $A_{y}=\hat{\Lambda}=E_{+}+E_{-} \partial_{y}$ which satisfies an important property $\hat{\Lambda}^{2}=\partial_{y}$. Choose the bare potentials $A_{k}$ from the SDYM hierarchy to be $\hat{\Lambda} \partial_{y}^{k-1}=\hat{\Lambda}^{2 k-1}, k \geqq 2$; then the SDYM hierarchy reduces to the higher KP flows expressed as

$$
\partial_{t_{k}} U=\partial_{x} \Phi_{+}^{(k)}-\left[U+E_{-} \partial_{y}, \Phi_{+}^{(k)}\right],
$$

where

$$
\Phi^{(k)}=W \hat{\Lambda}^{2 k-1} W^{-1}=W \hat{\Lambda} \partial_{y}^{k-1} W^{-1}=\sum_{l=0}^{\infty} \Phi_{l}^{(k)} \partial_{y}^{k-l}, \quad \Phi_{0}^{(k)}=E_{-} .
$$

Note that the right-hand side of (50) has order zero, i.e. is a function since the higher coefficients of $\partial_{y}$ vanish due to the recurrence relation for the $\Phi_{l}^{(k)}$. These higher KP flows are generalizations of higher KdV flows. However, they do not constitute the complete KP hierarchy as there exists another set of flows. To obtain them from our scheme, note that (50) deals with

$$
\Phi^{(k)}=\Phi^{2 k-1}, \quad \Phi=W \hat{\Lambda} W^{-1} .
$$

Now consider even powers of $\Phi$ :

$$
\tilde{\Phi}^{(k)}=\Phi^{2 k}=W \partial_{y}^{k} W^{-1}=\sum_{l=0}^{\infty} \tilde{\Phi}_{l}^{(k)} \partial_{y}^{k-l}, \quad \tilde{\Phi}_{0}^{(k) .}=\mathbf{1}
$$


with the corresponding flows (which become trivial in the $\mathrm{KdV}$ case) given by

$$
\partial_{\tilde{t}_{k}} U=\partial_{x} \tilde{\Phi}_{+}^{(k)}-\left[U+E_{-} \partial_{y}, \tilde{\Phi}_{+}^{(k)}\right],
$$

which, together with (50), constitute a commuting KP hierarchy.

Remark 27. For completeness one should include into this hierarchy linear equations $\partial_{t_{1}} u=\partial_{x} u$ and $\partial_{\tilde{t}_{1}} u=\partial_{y} u$, which can be obtained, respectively, from (50) and (51) by setting $k=1$.

Remark 28. Starting from our scheme one can recover Sato's approach [11] to the KP hierarchy. To make this connection more transparent, introduce $B_{k}=\left(\Phi^{k}\right)_{+}$ and $\tau_{k}=t_{(k-1) / 2}$ for odd $k$ and $\tau_{k}=\tilde{t}_{k / 2}$ for even $k$. Then $\Phi$ satisfies the differential equation

$$
\partial_{\tau_{k}} \Phi=\left[B_{k}, \Phi\right]
$$

and the KP hierarchy is given by

$$
\partial_{\tau_{k}} U=\partial_{x} B_{k}-\left[B_{1}, B_{k}\right] .
$$

These formulas are essentially the same as in [11] now written in "matrix form." More precisely, using the fact that linear equation (48) is equivalent to the " $y$-dependent" Schrödinger equation

$$
-\partial_{x}^{2} f+u f=-\partial_{y} f
$$

we can "trade off" $\partial_{y}$ in $W$ for $\partial_{x}$, so that from $W$ one can recover Sato's pseudodifferential operator in $\partial_{x}$. Recall that in Sect. 4.3 we used the same method to show how our approach coincides with the Gelfand-Dikii and Adler schemes.

Remark 29. Consider once again the differential equation

$$
\partial_{x} \Phi^{(k)}=\left[U+E_{-} \partial_{y}, \Phi^{(k)}\right] .
$$

Decomposing it into Cartan and nilpotent parts (cf. Sect. 4.3) we can obtain a recursion operator for the KP hierarchy. It gives a precise meaning to the formal expression $R$ and coincides with that obtained in [30]. In addition, considering the polynomial and Laurent parts with respect to $\partial_{y}$ of the above equation we get another form of the KP hierarchy

$$
\partial_{\tau_{k}} U=\operatorname{ad} E_{-}\left(\operatorname{Res} \Phi^{(k)}\right),
$$

where Res is the "non-commutative" residue - the coefficient in front of the $\partial_{y}^{-1}$ in the pseudodifferential operator $\Phi^{(k)}$.

Similarly, we note that one can also recover symmetries and conservation laws for the KP hierarchy.

\section{Concluding Remarks}

Thus we have seen how the general theory of intertwiners, developed for the SDYM system, produces, under reductions, many of the known results for integrable systems in $1+1$ and $2+1$ dimensions. For the $1+1$ case our approach yields, in addition to the hierarchies, conservation laws and symmetries, also a method of solution based on the underlying Riemann-Hilbert factorization problem. We expect that similar statements for the special SDYM system discussed 
in Sect. 5 will produce nonlocal Riemann-Hilbert and $\bar{\partial}$-problems for solving multidimensional systems.

An important feature of integrable systems which we did not address here is their Hamiltonian structure as a reduction of an appropriate "universal" structure for the SDYM equations. Considering several simple examples we conjecture that the underlying gradient flow structure of the SDYM equations may be exploited to generate the Hamiltonian structure for integrable equations. An intriguing problem in this direction is to understand possible connections between the "universal" SDYM structure and the formalism of classical $r$-matrix for $1+1$ integrable systems.

Acknowledgements. We appreciate useful discussions with Professor L. Faddeev at the early stage of this work. The work of M.J.A. is partially supported by the NSF, Grant DMS-9024528 and the Air Force Office of Scientific Research, Grant AFOSR-90-0039; L.T. is partially supported by the Paul and Gabriella Rosenbaum Foundation.

\section{References}

1. Chodos, A., Hadjimichael, E., Tze, C. (eds.), Solitons in Nuclear and Elementary Particle Physics. Proc. of the Lewes Workshop. Singapore: World Scientific 1984

2. Ashtekar, A.: Radiative degrees of freedom of the gravitational field in exact general relativity. J. Math. Phys. 22, 2885-2895 (1981)

3. Freed, David, S., Uhlenbeck, Karen, K.: Instantons and 4-manifolds. New York: Springer 1984

4a. Chau, Ling Lie: Integrability properties of supersymmetric Yang-Mills fields and relations with other nonlinear systems. Proc. of the $13^{\text {th }}$ Int. Coll. on Group Theoretical Methods in Physics, Zachary, W. W. (ed.), Singapore: World Scientific 1984

4b. Beals, R., Coifman, R.R.: The D-Bar approach to Inverse Scattering and Nonlinear Evolutions. Physica 18D, 242-249 (1986)

4c. Bruschi, M., Levi, D., Ragnisco, O.: Nonlinear Partial Differential Equation and Bäcklund Transformations Related to the 4-Dimensional Self-Dual Yang-Mills Equation. Lett. Nuovo Cimento 33, 263-266 (1982)

5. Ward, R.S.: Integrable and solvable systems and relations among them. Phil. Trans. Roy. Soc. Lond. A315, 451-457 (1985)

6. Atiyah, M.F.: Classical Geometry of Yang-Mills Fields. Fermi Lectures, Scuola Normale Pisa (1980)

7. Belavin, A.A., Zakharov, V.E.: Yang-Mills equations as inverse scattering problem. Phys. Lett. 73B, 53-57 (1978)

8. Atiyah, M.F., Ward, R.S.: Instantons and algebraic geometry. Commun. Math. Phys. 55, 117-124 (1977)

9. Schur, I.: Über vertauschbare lineare Differentialausdrücke. Gesammelte Abhandlungen I, 170-176, Springer 1905

10. Mulase, M.: KP equations, strings and Schottky problem. In: Algebraic Analysis, Kashiwara, M., Kawai, T. (eds.), II, 473-492, New York: Academic Press 1988

11. Sato, M., Sato, Y.: Soliton equations as dynamical systems on infinite dimensional Grassmann manifold. Lect. Notes in Num. Appl. Anal. 5, 259-271 (1982)

12. Faddeev, L., Takhtajan, L.: Hamiltonian Methods in the Theory of Solitons. Berlin, Heidelberg, New York: Springer 1987

13. Mason, J., Sparling, G.: Twistor correspondence for the soliton hierarchies. J. Geom. and Phys. 8, 243-271 (1992)

14. Prasad, M.K., Sinha, A., Chau-Wang, L.L.: Non-local continuity equation for self-dual $S U(N)$ Yang-Mills fields. Phys. Lett. 87B, 237-238 (1979)

15. Mason, J., Sparling, G.: Nonlinear Schrödinger and Korteweg-de Vries equations are reductions of self-dual Yang-Mills equations. Phys. Lett. 137A, 29-33 (1989) 
16. Zakharov, V.E., Manakov, S.V.: The theory of resonant interaction of wave packets in nonlinear media. Sov. Phys. JETP Lett. 18, 243-245 (1973)

17. Zakharov, V.E., Novikov, S.P., Manakov, S.V., Pitaevsky, L.P.: Theory of Solitons: The Inverse Scattering Method. New York: Consultant Bureau 1984

18. Ablowitz, M.J., Segur, H.: Solitons and Inverse Scattering Transform. Philadelphia: SIAM 1981

19. Chakravarty, S., Ablowitz, M.J.: On reduction of self-dual Yang-Mills equations. In: Painleve Transcendents, Their Asymptotics and Physical Applications. Levi, D., Winternitz, P. (eds.) London, New York: Plenum 1992

20. McIntosh, I.: Soliton equations and connections with self-dual curvature. Preprint (1991)

21. Zakharov, V.E., Shabat, A.B.: Exact theory of two-dimensional self-focusing and one-dimensional self-modulation of waves in non-linear media. Sov. Phys. JETP 34, 62-69 (1972)

22. Gelfand, I.M., Dikii, L.A.: Asymptotic behaviour of the resolvent of Sturm-Liouville equations and the algebra of the Korteweg-de Vries equations. Russ. Math. Surv. 30:5, 77-113 (1975)

23. Adler, M.: On a trace functional for formal pseudodifferential operators and symplectic structure of the Korteweg-de Vries type equations. Invent. Math. 50, 219-248 (1979)

24. Hermite, Ch.: Sur l'équation de Lamé. - Cours d'analyse de l'École Polytechn. Paris, 1872-1873, 32-e leçon; Oeuvres, III, 118-122, Paris 1912

25. Gardner, C.S., Greene, J.M., Kruskal, M.D., Miura, R.H.: Korteweg-de Vries equation and generalizations. VI. Methods for exact solution. Commun. Pure Appl. Math. 27, 97-133 (1974)

26. Ablowitz, M.J., Kaup, D.J., Newell, A.C., Segur, H.: The inverse scattering transform-Fourier analysis for nonlinear problems. Stud. Appl. Math. 53, 249-315 (1974)

27. Ablowitz, M.J., Haberman, R.: Nonlinear evolution equations - two and three dimensions. Phys. Rev. Lett. 35, 1185-1188 (1975)

28. Zakharov, V.E., Shabat, A.B.: Integration of nonlinear equations of mathematical physics by the method of inverse scattering, II. Func. Anal. Appl. 13, 166-176 (1979)

29. Ablowitz, M.J., Clarkson, P.A.: Solitons, Nonlinear Evolution Equations and Inverse Scattering. London Mathematical Society Lecture Note Series, 149, Cambridge: Cambridge University Press 1992

30. Fokas, A.S., Gel'fand, I.M.: Bi-Hamiltonian Structures and Integrability. Preprint, INS 188 (1992)

31. Konopelchenko, B.G.: Nonlinear Integrable Equations: Recursion Operators, Group-Theoretical and Hamiltonian Structures of Soliton Equations. Lect. Notes in Physics, 270, Berlin, Heidelberg, New York: Springer 1987

32. Fokas, A.S., Santini, P.M.: Recursion operators and bi-Hamiltonian structures in multidimensions, II. Commun. Math. Phys. 116, 449-474 (1988)

Communicated by N.Yu. Reshetikhin 